1 Hacettepe Journal of Mathematics and Statistics

$\bigcap$ Volume 47 (6) (2018), 1503-1511

\title{
Distinguishability of a source function in a time fractional inhomogeneous parabolic equation with Robin boundary condition
}

\author{
Ebru Ozbilge*† and Ali Demir ${ }^{\ddagger}$
}

\begin{abstract}
This article deals with the mathematical analysis of the inverse problem of identifying the distinguishability of input-output mappings in the linear time fractional inhomogeneous parabolic equation $\mathrm{D}_{t}^{\alpha} u(x, t)=$ $\left(k(x) u_{x}\right)_{x}+F(x, t) \quad 0<\alpha \leq 1$, with Robin boundary conditions $u(0, t)=\psi_{0}(t), u_{x}(1, t)=\gamma\left(u(1, t)-\psi_{1}(t)\right)$. By defining the inputoutput mappings $\Phi[\cdot]: \mathcal{K} \rightarrow C^{1}[0, T]$ and $\Psi[\cdot]: \mathcal{K} \rightarrow C[0, T]$ the inverse problem is reduced to the problem of their invertibility. Hence, the main purpose of this study is to investigate the distinguishability of the inputoutput mappings $\Phi[\cdot]$ and $\Psi[\cdot]$. Moreover, the measured output data $f(t)$ and $h(t)$ can be determined analytically by a series representation, which implies that the input-output mappings $\Phi[\cdot]: \mathcal{K} \rightarrow C^{1}[0, T]$ and $\Psi[\cdot]: \mathcal{K} \rightarrow C[0, T]$ can be described explicitly.
\end{abstract}

Keywords: Inverse problem, time-fractional parabolic equation, distinguishability.

Mathematics Subject Classification (2010): 31B20,65L09, 35R11

Received: 03.11.2015 Accepted : 04.10.2016 Doi : 10.15672/HJMS.20164517213

*Department of Mathematics, Faculty of Science and Literature, Izmir University of Economics, Izmir, Turkey, Email: ebruozbilge@gmail.com

${ }^{\dagger}$ Corresponding Author.

${ }^{\ddagger}$ Department of Mathematics, Kocaeli University, Umuttepe, 41380 Izmit - Kocaeli, Turkey, Email: ademir@kocaeli.edu.tr 


\section{Introduction}

The inverse problem of unknown source function in a linear inhomogeneous parabolic equation by using overmeasured data has generated an increasing amount of interest from engineers and scientist during the last few decades. This kind of problems play a crucial role in engineering, physics and applied mathematics. The problem of recovering unknown source function in the mathematical model of a physical phenomena is frequently encountered.

Intensive study has been carried out on this kind of problem, and various inverse problems and many numerical methods developed [1]-[3], [8]-[14] and [18]-[21].

In papers [29]-[31] a coupled method for inverse source problem of spatial fractional anomalous diffusion equations, a boundary-type collocation method for inverse Cauchy inhomogeneous potential problems were considered. Moreover, inverse problems for a time fractional parabolic equations with various boundary conditions has been studied by Ozbilge and Demir [15],[24]-[26].

The existence and uniqueness of solutions for fractional differential equations with nonlocal and integral boundary conditions have been studied by Ashyralyev and Sharifov [7]. Initial-boundary value problems for the one dimensional time fractional diffusion equation was studied by Amanov and Ashyralyev in [1], finite difference method for fractional parabolic equations with Neumann boundary conditions was studied by Ashyralyev and Cakir in [4], numerical solution of fractional Schrodinger differential equation with Dirichlet boundary condition was studied by Ashyralyev and Hicdurmaz in [6]. Moreover, Ashyralyev and Dal studied finite difference methods and iteration methods for fractional hyperbolic partial equations with Neumann condition in [5]. Second order implicit finite difference schemes have been applied to the right-hand side of the identification problem by Erdogan and Ashyralyev [17].

Fractional differential equations are generalizations of ordinary and partial differential equations to an arbitrary fractional order. By linear time-fractional parabolic equation, we mean certain parabolic-like partial differential equation governed by master equations containing fractional derivatives in time. The research areas of fractional differential equations range from the theoretical to the applied aspects.

The main goal of this study is to investigate the distinguishability of the unknown source function via input-output mappings in a one dimensional time fractional inhomogeneous parabolic equation. We first obtain the unique solution of this problem using Fourier method of separation of variables with respect to the eigenfunctions of a corresponding Sturm-Liouville eigenvalue problem under certain conditions [15]. As the next step, the noisy free measured output data $f(t)$ and $h(t)$ are used to introduce the input-output mappings $\Phi[\cdot]: \mathcal{K} \rightarrow C^{1}[0, T]$ and $\Psi[\cdot]: \mathcal{K} \rightarrow C[0, T]$ where $\mathcal{K}$ represents the set of admissible source functions. Finally we investigate the distinguishability of the unknown source function $F(x, t)$ via the above input-output mappings $\Phi[\cdot]$ and $\Psi[\cdot]$.

Consider now the following initial boundary value problem:

$$
\left\{\begin{array}{l}
D_{t}^{\alpha} u(x, t)=\left(k(x) u_{x}\right)_{x}+F(x, t) \quad, \quad 0<\alpha \leq 1 \quad, \quad(x, t) \in \Omega_{T} \\
u(x, 0)=g(x), \quad 0<x<1, \\
u(0, t)=\psi_{0}(t), \quad u_{x}(1, t)=\gamma\left(u(1, t)-\psi_{1}(t)\right), \quad 0<t<T
\end{array}\right.
$$

where $\Omega_{T}=\left\{(x, t) \in R^{2}: 0<x<1,0<t \leq T\right\}$ and the fractional derivative $D_{t}^{\alpha} u(x, t)$ is defined in the Caputo-Dzherbashyan sense $D_{t}^{\alpha} u(x, t)=\left(I^{1-\alpha} u^{\prime}\right)(t), \quad 0<\alpha \leq 1, I^{\alpha}$ being the Riemann-Liouville fractional integral

$$
\left(I^{\alpha} f\right)(t)=\left\{\begin{array}{cc}
\frac{1}{\Gamma(\alpha)} \int(t-\tau)^{\alpha-1} f(\tau) d \tau & , 0<\alpha \leq 1 \\
f(t), & \alpha=0
\end{array}\right.
$$


The left and right boundary value functions $\psi_{0}(t)$ and $\psi_{1}(t)$ belong to $C[0, T]$. The functions $0<c_{0} \leq k(x)<c_{1}$ and $g(x)$ satisfy the following conditions:

(C1) $k(x) \in C^{1}[0,1]$

(C2) $g(x) \in C^{2}[0,1], g(0)=\psi_{0}(0), \quad g^{\prime}(1)=\gamma\left(g(1)-\psi_{1}(0)\right)$.

Under these conditions, the initial boundary value problem (1.1) has the unique solution $u(x, t)$ defined in the domain $\bar{\Omega}_{T}=\left\{(x, t) \in \mathbb{R}^{2}: 0 \leq x \leq 1,0 \leq t \leq T\right\}$ which belongs to the space $C\left(\bar{\Omega}_{T}\right) \cap W_{t}^{1}(0, T] \cap C_{x}^{2}(0,1)$ where $C\left(\bar{\Omega}_{T}\right)=\left\{f(\cdot, \cdot): \bar{\Omega}_{T} \rightarrow \mathbb{R}\right.$ : $f$ is continuous $\}, W_{t}^{1}(0, T]=\left\{f(x, \cdot):(0, T] \rightarrow \mathbb{R}: f(x, \cdot), f_{t}(x, \cdot) \in L^{2}[0, T]\right\}$ and $C_{x}^{2}(0,1)=\left\{f(\cdot, t):(0,1) \rightarrow \mathbb{R}: f(\cdot, t), f_{x}(\cdot, t), f_{x x}(\cdot, t)\right.$ are continuous $\}$. Moreover, it satisfies the equation, initial and boundary conditions. The space $W_{t}^{1}(0, T]$ contains the functions $f \in C^{1}(0, T]$ such that $f^{\prime}(x) \in L(0, T)$.

This kind of problems play a crucial role in engineering, physics and applied mathematics since it is used successfully to model complex phenomenian various fields such as fluid mechanics, viscoelasticity, physics, chemistry and engineering.

Consider the inverse problem of determining the distinguishability of the unknown source function $F(x, t)$ from the mixed type of measured output data at the boundaries $x=0$ and $x=1$ :

$$
\begin{gathered}
\Phi[F]=\left.k(x) u_{x}(x, t ; F)\right|_{x=0}, \quad F \in \mathcal{K} \subseteq C^{1}\left(\Omega_{T}\right) \\
\Psi[F]=\left.u(x, t ; F)\right|_{x=1}, \quad F \in \mathcal{K} \subseteq C\left(\Omega_{T}\right) .
\end{gathered}
$$

Then, the inverse problem with the measured output data $f(t)$ and $h(t)$ can be formulated as follows:

$$
\begin{aligned}
& \Phi[F]=f, \quad f \in C^{1}(0, T] \\
& \Psi[F]=h, \quad h \in C(0, T] .
\end{aligned}
$$

These formulations reduce the inverse problem of determining unknown source function $F(x, t)$ to the problem of invertibility of the input-output mappings $\Phi[\cdot]$ and $\Psi[\cdot]$. Hence this leads us to study the distinguishability of the source function via the above inputoutput mappings. We say that the mappings $\Phi[\cdot]: \mathcal{K} \rightarrow C^{1}[0, T]$ and $\Psi[\cdot]: \mathcal{K} \rightarrow C[0, T]$ have the distinguishability property if $\Phi\left[F_{1}\right] \neq \Phi\left[F_{2}\right]$ implies $F_{1}(x, t) \neq F_{2}(x, t)$ and $\Psi\left[F_{1}\right] \neq \Psi\left[F_{2}\right]$ implies $F_{1}(x, t) \neq F_{2}(x, t)$. This, in particular, means injectivity of the inverse mappings $\Phi^{-1}$ and $\Psi^{-1}$. In this paper, measured output data of Neumann type at the boundary $x=0$ and measured output data of Dirichlet type at the boundary $x=1$ are used in the investigation of the distinguishability of the unknown source function $F(x, t)$ via the above input-output mappings. As well as this, analytical results are obtained for the measured output data.

The paper is organized as follows. In section 2, an analysis of the inverse problem with the single measured output data $f(t)$ at the boundary $x=0$ is given. An analysis of the inverse problem with the single measured output data $h(t)$ at the boundary $x=1$ is considered in section 3. Finally, some concluding remarks are given in the last section.

\section{An analysis of the inverse problem with given measured data $f(t)$}

Consider now the inverse problem with one measured output data $f(t)$ at $x=0$. In order to formulate the solution of the parabolic problem (1.1) by using Fourier method of the separation of variables, let us first introduce an auxiliary function $v(x, t)$ as follows:

$$
v(x, t)=u(x, t)-\psi_{0}(t)-\gamma\left(u(1, t)-\psi_{1}(t)\right) x, \quad x \in[0,1],
$$


by which we transform the problem (1.1) into a problem with homogeneous boundary conditions. Hence the initial boundary value problem (1.1) can be rewritten in terms of $v(x, t)$ in the following form:

$$
\left\{\begin{array}{l}
D_{t}^{\alpha} v(x, t)-v_{x x}(x, t)=\left((k(x)-1) v_{x}(x, t)\right)_{x}+k^{\prime}(x) \gamma\left(u(1, t)-\psi_{1}(t)\right) \\
-D_{t}^{\alpha} \psi_{0}(t)-D_{t}^{\alpha} \gamma\left(u(1, t)-\psi_{1}(t)\right) x+F(x, t), \\
v(x, 0)=g(x)-\psi_{0}(0)-\gamma\left(u(1,0)-\psi_{1}(0)\right) x, \quad 0<x<1, \\
v(0, t)=0, \quad v_{x}(1, t)=0, \quad 0<t<T .
\end{array}\right.
$$

The unique solution of the initial-boundary value problem can be represented in the following form [15]:

$$
\begin{gathered}
v(x, t)=\sum_{n=1}^{\infty}<\zeta(\theta), \phi_{n}(\theta)>E_{\alpha, 1}\left(-\lambda_{n} t^{\alpha}\right) \phi_{n}(x) \\
+\sum_{n=1}^{\infty}\left(\int_{0}^{t} s^{\alpha-1} E_{\alpha, \alpha}\left(-\lambda_{n} s^{\alpha}\right)\left(<\xi(\theta, t-s), \phi_{n}(\theta)>+<F(\theta, t-s), \phi_{n}(\theta)>\right) d s\right) \phi_{n}(x)
\end{gathered}
$$

where

$$
\zeta(x)=g(x)-\psi_{0}(0)-\gamma\left(u(1,0)-\psi_{1}(0)\right) x,
$$

$\xi(x, t)=\left((k(x)-1) v_{x}(x, t)\right)_{x}+k^{\prime}(x) \gamma\left(u(1, t)-\psi_{1}(t)\right)-D_{t}^{\alpha} \psi_{0}(t)-D_{t}^{\alpha} \gamma\left(u(1, t)-\psi_{1}(t)\right) x$,

Moreover $\left\langle\zeta(\theta), \phi_{n}(\theta)>=\int_{0}^{1} \phi_{n}(\theta) \zeta(\theta) d \theta, E_{\alpha, \beta}\right.$ being the generalized Mittag-Leffler function defined by

$$
E_{\alpha, \beta}(z)=\sum_{n=0}^{\infty} \frac{z^{n}}{\Gamma(\beta n+\alpha)} .
$$

Assume that $\phi_{n}(x)$ is the solution of the following Sturm-Liouville problem:

$$
\left\{\begin{array}{l}
-\phi_{x x}(x)=\lambda \phi(x), \quad 0<x<1 \\
\phi(0)=0, \quad \phi_{x}(1)=0, \quad 0<t<T,
\end{array}\right.
$$

The Neumann type of measured output data at the boundary $x=0$ in terms of $v(x, t)$ can be written in the following form:

$$
k(0)\left(v_{x}(0, t)+\gamma\left(u(1, t)-\psi_{1}(t)\right)=f(t), \quad t \in(0, T]\right.
$$

In order to arrange the above solution, let us define the followings:

$$
\begin{gathered}
z_{n}(t)=<\zeta(\theta), \phi_{n}(\theta)>E_{\alpha, 1}\left(-\lambda_{n} t^{\alpha}\right), \\
w_{n}(t)=\int_{0}^{t} s^{\alpha-1} E_{\alpha, \alpha}\left(-\lambda_{n} s^{\alpha}\right)<\xi(\theta, t-s), \phi_{n}(\theta)>d s, \\
y_{n}(t)=\int_{0}^{t} s^{\alpha-1} E_{\alpha, \alpha}\left(-\lambda_{n} s^{\alpha}\right)<F(\theta, t-s), \phi_{n}(\theta)>d s .
\end{gathered}
$$

The solution in terms of $z_{n}(t), w_{n}(t)$ and $y_{n}(t)$ can then be rewritten in the following form:

$$
v(x, t)=\sum_{n=1}^{\infty} z_{n}(t) \phi_{n}(x)+\sum_{n=1}^{\infty} w_{n}(t) \phi_{n}(x)+\sum_{n=1}^{\infty} y_{n}(t) \phi_{n}(x) .
$$

Differentiating both sides of the above identity with respect to $x$ and substituting $x=0$ yields:

$$
v_{x}(0, t)=\sum_{n=1}^{\infty} z_{n}(t) \phi_{n}^{\prime}(0)+\sum_{n=1}^{\infty} w_{n}(t) \phi_{n}^{\prime}(0)+\sum_{n=1}^{\infty} y_{n}(t) \phi_{n}^{\prime}(0) .
$$


Taking into account the over-measured data $k(0)\left(v_{x}(0, t)+\gamma\left(u(1, t)-\psi_{1}(t)\right)=f(t)\right.$

$$
\begin{aligned}
& f(t)=k(0)\left(\gamma\left(u(1, t)-\psi_{1}(t)\right)+\sum_{n=1}^{\infty} z_{n}(t) \phi_{n}^{\prime}(0)+\sum_{n=1}^{\infty} w_{n}(t) \phi_{n}^{\prime}(0)\right. \\
& \left.+\sum_{n=1}^{\infty} y_{n}(t) \phi_{n}^{\prime}(0)\right),
\end{aligned}
$$

is obtained, which implies that $f(t)$ can be determined analytically. The right-hand side of identity (2.4) defines the input-output mapping $\Phi[F]$ on the set of admissible source function $\mathcal{K}$ :

$$
\begin{aligned}
& \Phi[F](t):=k(0)\left(\gamma\left(u(1, t)-\psi_{1}(t)\right)+\sum_{n=1}^{\infty} z_{n}(t) \phi_{n}^{\prime}(0)+\sum_{n=1}^{\infty} w_{n}(t) \phi_{n}^{\prime}(0)\right. \\
& \left.+\sum_{n=1}^{\infty} y_{n}(t) \phi_{n}^{\prime}(0)\right), \quad \forall t \in[0, T] .
\end{aligned}
$$

The following lemma implies the relation between the source functions $F_{1}(x, t), F_{2}(x, t) \in$ $\mathcal{K}$ at $x=0$ and the corresponding outputs $f_{j}(t):=k(0) u_{x}\left(0, t ; F_{j}\right), j=1,2$.

2.1. Lemma. Let $v_{1}(x, t)=v\left(x, t ; F_{1}\right)$ and $v_{2}(x, t)=v\left(x, t ; F_{2}\right)$ be the solutions of the direct problem (2.1), corresponding to the admissible source functions $F_{1}(x, t), F_{2}(x, t) \in$ $\mathcal{K}$. If $f_{j}(t)=k(0)\left(v_{x}\left(0, t ; F_{j}\right)+\gamma\left(u\left(1, t ; F_{j}\right)-\psi_{1}(t)\right), j=1,2\right.$, are the corresponding outputs. The outputs $f_{j}(t), j=1,2$, satisfy the following integral identity:

$$
\Delta f(t)=k(0)\left(\sum_{n=1}^{\infty} \Delta z_{n}(t) \phi_{n}^{\prime}(0)+\sum_{n=1}^{\infty} \Delta w_{n}(t) \phi_{n}^{\prime}(0)+\sum_{n=1}^{\infty} \Delta y_{n}(t) \phi_{n}^{\prime}(0)+\gamma \Delta u_{n}(1, t)\right)
$$

for each $t \in(0, T]$ where $\Delta f(t)=f_{1}(t)-f_{2}(t), \Delta w_{n}(t)=w_{n}^{1}(t)-w_{n}^{2}(t), \Delta z_{n}(t)=z_{n}^{1}(t)-$ $z_{n}^{2}(t), \Delta F(x, t)=F_{1}(x, t)-F_{2}(x, t)$ and $\Delta y_{n}(t)=y_{n}^{1}(t)-y_{n}^{2}(t)=\int_{0}^{t} s^{\alpha-1} E_{\alpha, \alpha}\left(-\lambda_{n} s^{\alpha}\right)<$ $\Delta F(\theta, t-s), \phi_{n}(\theta)>d s$.

Proof. By using identity (2.4), the measured output data $f_{j}(t):=k(0)\left(v_{x}^{j}(0, t)+\gamma(u(1, t)-\right.$ $\left.\psi_{1}(t)\right), j=1,2$ can be written as follows:

$$
\begin{aligned}
& f_{1}(t)=k(0)\left(\gamma\left(u_{n}^{1}\left(1, t ; F_{1}\right)-\psi_{1}(t)\right)+\sum_{n=1}^{\infty} z_{n}^{1}(t) \phi_{n}^{\prime}(0)+\sum_{n=1}^{\infty} w_{n}^{1}(t) \phi_{n}^{\prime}(0)+\sum_{n=1}^{\infty} y_{n}^{1}(t) \phi_{n}^{\prime}(0)\right), \\
& f_{2}(t)=k(0)\left(\gamma\left(u_{n}^{2}\left(1, t ; F_{2}\right)-\psi_{1}(t)\right)+\sum_{n=1}^{\infty} z_{n}^{2}(t) \phi_{n}^{\prime}(0)+\sum_{n=1}^{\infty} w_{n}^{2}(t) \phi_{n}^{\prime}(0)+\sum_{n=1}^{\infty} y_{n}^{2}(t) \phi_{n}^{\prime}(0)\right)
\end{aligned}
$$

respectively. Hence the difference of these formulas implies the desired result.

The lemma and the definitions enable us to reach the following conclusion:

2.2. Corollary. Let the conditions of Lemma 2.1 hold. If in addition

$$
<F_{1}(x, t)-F_{2}(x, t), \phi_{n}(x)>=0
$$

and

$$
\Delta u(1, t)=u_{n}^{1}\left(x, t ; F_{1}\right)-u_{n}^{2}\left(x, t ; F_{2}\right)=0,
$$

$\forall t \in(0, T], \quad \forall n=0,1, \ldots$ hold, then $f_{1}(t)=f_{2}(t), \forall t \in(0, T]$. 
Note that $F_{1}(x, t) \neq F_{2}(x, t)$ implies $<F_{1}(x, t)-F_{2}(x, t), \phi_{n}(x)>\neq 0$ and $\Delta y_{n}(t) \neq 0$, $\forall n=0,1,2, \ldots$ Moreover $\Delta u(1, t) \neq 0$ implies that $\Delta z_{n}(t) \neq 0$ and $\Delta w_{n}(t) \neq 0$, $\forall n=0,1,2, \ldots$. Hence by Lemma 2.1 we conclude that $f_{1}(t) \neq f_{2}(t) \forall t \in(0, T]$. Moreover, it leads us to the following important consequence that the input-output mapping $\Phi[F]$ is distinguishable, i.e., $F_{1}(x, t) \neq F_{2}(x, t)$ implies $\Phi\left[F_{1}\right] \neq \Phi\left[F_{2}\right]$.

2.3. Theorem. Let conditions (C1),(C2) hold. Assume that $\Phi[\cdot]: \mathcal{K} \rightarrow C^{1}[0, T]$ is the input-output mapping defined by (2.5) and corresponding to the measured output $f(t):=k(0) u_{x}(0, t)$. In this case the mapping $\Phi[F]$ has the distinguishability property in the class of admissible parameters $\mathcal{K}$, i.e.,

$$
\Phi\left[F_{1}\right] \neq \Phi\left[F_{2}\right] \quad \forall F_{1}, F_{2} \in \mathcal{K}, \quad F_{1}(x, t) \neq F_{2}(x, t) .
$$

\section{An analysis of the inverse problem with given measured data} $h(t)$

Consider now the inverse problem with one measured output data $h(t)$ at $x=1$. Taking into account the over-measured data $h(t)=\left(v(1, t)+\psi_{0}(t)+\gamma\left(u(1, t)-\psi_{1}(t)\right)\right.$

$$
\begin{aligned}
& h(t)=\left(\psi_{0}(t)+\gamma\left(u(1, t)-\psi_{1}(t)\right)+\sum_{n=1}^{\infty} z_{n}(t) \phi_{n}(1)\right. \\
& \left.+\sum_{n=1}^{\infty} w_{n}(t) \phi_{n}(1)+\sum_{n=1}^{\infty} y_{n}(t) \phi_{n}(1)\right),
\end{aligned}
$$

is obtained which implies that $h(t)$ can be determined analytically. The right-hand side of identity (3.1) defines the input-output mapping $\Psi[F]$ on the set of admissible source functions $\mathcal{K}$ :

$$
\begin{aligned}
& \Psi[F](t):=\left(\psi_{0}(t)+\gamma\left(u(1, t)-\psi_{1}(t)\right)+\sum_{n=1}^{\infty} z_{n}(t) \phi_{n}(1)\right. \\
& \left.+\sum_{n=1}^{\infty} w_{n}(t) \phi_{n}(1)+\sum_{n=1}^{\infty} y_{n}(t) \phi_{n}(1)\right) .
\end{aligned}
$$

The following lemma implies the relation between the parameters $F_{1}(x, t), F_{2}(x, t) \in \mathcal{K}$ at $x=1$ and the corresponding outputs $h_{j}(t):=u\left(1, t ; F_{j}\right), j=1,2$.

3.1. Lemma. Let $v_{1}(x, t)=v\left(x, t ; F_{1}\right)$ and $v_{2}(x, t)=v\left(x, t ; F_{2}\right)$ be the solutions of the direct problem (2.1), corresponding to the admissible source functions $F_{1}(x, t), F_{2}(x, t) \in$ $\mathcal{K}$. If $h_{j}(t)=v\left(1, t ; F_{j}\right)+\psi_{0}(t)+\gamma\left(u\left(1, t ; F_{j}\right)-\psi_{1}(t)\right), j=1,2$, are the corresponding outputs. The outputs $h_{j}(t), j=1,2$, satisfy the following integral identity:

$$
\Delta h(t)=\left(\sum_{n=1}^{\infty} \Delta z_{n}(t) \phi_{n}(1)+\sum_{n=1}^{\infty} \Delta w_{n}(t) \phi_{n}(1)+\sum_{n=1}^{\infty} \Delta y_{n}(t) \phi_{n}(1)+\gamma \Delta u_{n}(1, t)\right),
$$

for each $t \in(0, T]$ where $\Delta h(t)=h_{1}(t)-h_{2}(t), \Delta w_{n}(t)=w_{n}^{1}(t)-w_{n}^{2}(t), \Delta z_{n}(t)=z_{n}^{1}(t)-$ $z_{n}^{2}(t), \Delta F(x, t)=F_{1}(x, t)-F_{2}(x, t)$ and $\Delta y_{n}(t)=y_{n}^{1}(t)-y_{n}^{2}(t)=\int_{0}^{t} s^{\alpha-1} E_{\alpha, \alpha}\left(-\lambda_{n} s^{\alpha}\right)<$ $\Delta F(\theta, t-s), \phi_{n}(\theta)>d s$.

Proof. By using identity (3.1), the measured output data $h_{j}(t):=v\left(1, t ; F_{j}\right)+\psi_{0}(t)+$ $\gamma\left(u\left(1, t ; F_{j}\right)-\psi_{1}(t)\right) x, j=1,2$ can be written as follows:

$$
h_{1}(t)=\psi_{0}(t)+\gamma\left(u_{n}^{1}\left(1, t ; F_{1}\right)-\psi_{1}(t)\right)+\sum_{n=1}^{\infty} z_{n}^{1}(t) \phi_{n}(1)+\sum_{n=1}^{\infty} w_{n}^{1}(t) \phi_{n}(1)+\sum_{n=1}^{\infty} y_{n}^{1}(t) \phi_{n}(1) \text {, }
$$


$h_{2}(t)=\psi_{0}(t)+\gamma\left(u_{n}^{2}\left(1, t ; F_{2}\right)-\psi_{1}(t)\right)+\sum_{n=1}^{\infty} z_{n}^{2}(t) \phi_{n}(1)+\sum_{n=1}^{\infty} w_{n}^{2}(t) \phi_{n}(1)+\sum_{n=1}^{\infty} y_{n}^{2}(t) \phi_{n}(1)$,

respectively. Hence the difference of these formulas implies the desired result.

3.2. Corollary. Let the conditions of Lemma 3.1 hold. If in addition

$$
<F_{1}(x, t)-F_{2}(x, t), \phi_{n}(x)>=0,
$$

and

$$
\Delta u(1, t)=u_{n}^{1}\left(x, t ; F_{1}\right)-u_{n}^{2}\left(x, t ; F_{2}\right)=0,
$$

$\forall t \in(0, T], \quad \forall n=0,1, \ldots$ hold, then $h_{1}(t)=h_{2}(t), \forall t \in(0, T]$.

Note that $F_{1}(x, t) \neq F_{2}(x, t)$ implies $<F_{1}(x, t)-F_{2}(x, t), \phi_{n}(x)>\neq 0$ and $\Delta y_{n}(t) \neq 0$, $\forall n=0,1,2, \ldots$. Moreover $\Delta u(1, t) \neq 0$ implies that $\Delta z_{n}(t) \neq 0$ and $\Delta w_{n}(t) \neq 0$, $\forall n=0,1,2, \ldots$. Hence by Lemma 3.1 we conclude that $h_{1}(t) \neq h_{2}(t) \forall t \in(0, T]$. Moreover, it leads us to the following important consequence that the input-output mapping $\Psi[F]$ is distinguishable, i.e., $F_{1}(x, t) \neq F_{2}(x, t)$ implies $\Psi\left[F_{1}\right] \neq \Psi\left[F_{2}\right]$.

3.3. Theorem. Let conditions (C1),(C2) hold. Assume that $\Psi[\cdot]: \mathcal{K} \rightarrow C[0, T]$ is the input-output mapping defined by (3.2) and corresponding to the measured output $h(t):=u\left(1, t ; F_{j}\right), \quad j_{1,2}$. In this case the mapping $\Psi[F]$ has the distinguishability property in the class of admissible parameters $\mathcal{K}$, i.e.,

$\Psi\left[F_{1}\right] \neq \Psi\left[F_{2}\right] \quad \forall F_{1}, F_{2} \in \mathcal{K}$, implies $\quad F_{1}(x, t) \neq F_{2}(x, t)$.

\section{Conclusion}

The aim of this study was to investigate the distinguishability properties of the inputoutput mappings $\Phi[\cdot]: \mathcal{K} \rightarrow C^{1}[0, T]$ and $\Psi[\cdot]: \mathcal{K} \rightarrow C[0, T]$, which are determined by the measured output data at $x=0$ and $x=1$, respectively. In this study, we conclude that the distinguishability of the input-output mappings hold which implies the injectivity of the inverse mappings $\Phi^{-1}$ and $\Psi^{-1}$. The measured output data $f(t)$ and $h(t)$ are obtained analytically by a series representation, which leads to the explicit form of the input-output mappings $\Phi[\cdot]$ and $\Psi[\cdot]$. This work advances our understanding of the use of the Fourier method of separation of variables and the input-output mapping in the investigation of inverse problems for fractional parabolic equations. The author plans to consider various fractional inverse problems in future studies, since the method discussed has a wide range of applications.

Acknowledgements The research was supported by parts by the Scientific and Technical Research Council (TUBITAK) of Turkey and Izmir University of Economics.

\section{References}

[1] Amanov, D., Ashyralyev, A., Initial-boundary value problem for fractional partial differential equations of higher order, Abstr. Appl. Anal. 2012, Article Id: 9733102, 470-484, 2012.

[2] Ashyralyev, A., A note on fractional derivatives and fractional powers of operators, J. Math. Anal. Appl. 357, 232-236, 2009.

[3] Ashyralyev, A., Artykov, M., Cakir, Z., A note on fractional parabolic differential and difference equations, AIP Conf. Proceedings 1611, 251-254, 2014.

[4] Ashyralyev, A., Cakir, Z., FDM for fractional parabolic equations with the Neumann condition, Adv. Differential Equations, 120, Doi:10.1186/1687-1847-2013-120, 2013. 
[5] Ashyralyev, A., Dal, F., Finite difference and iteration methods for fractional hyperbolic partial differential equations with neumann condition, Discrete Dyn. Nat. Soc. 2012, Article Id: $434976,2012$.

[6] Ashyralyev, A., Hicdurmaz,B., On the numerical solution of fractional schrodinger differential equations with dirichlet condition, Int. J. Comput. Math., 89, 1927-1936, 2012.

[7] Ashyralyev, A., Sharifov,Y.A., Existence and Uniqueness of solutions for the system of nonlinear fractional differential equations with nonlocal and integral boundary conditions, Abst. and Appl.Analys., 2012, Article Id: 594802,2012.

[8] Baglan, I., Determination of a coefficient in a quasilinear parabolic equation with periodic boundary condition, Inv. Probl.in Sci.and Eng., 23 (5), 884-900, 2015.

[9] Baglan, I., Kanca, F., Weak generalized and numerical solution for a quasilinear pseudoparabolic equation with nonlocal boundary condition, Adv. Differential Equations, 2014 (277), Doi:10.1186/1687-1847-2014-277, 2014.

[10] Baglan, I., Kanca, F., An inverse coefficient problem for a quasilinear parabolic equation with periodic boundary and integral overdetermination condition, Math. Meths. in the Appl. Sci., 38 (5), 851-867, 2015.

[11] Cannon, J.R., Lin, Y. An inverse problem fo finding a parameter in a semi-linear heat equation, J. Math. Anal. Appl. 145 (1), 470-484, 1990.

[12] Cannon, J. R., Lin, Y. Determination of source parameter in parabolic equations, Mechanica. 27, 85-94, 1992.

[13] Cannon, J.R., Lin, Y. Determination of a parameter $p(t)$ in some quasi-linear parabolic differential equations, Inv. Prob. 4, 35-44, 1998.

[14] Dehghan, M. Identification of a time-dependent coefficient in a partial differential equation subject to an extra measurement, Numer. Meth. Part. Diff. Eq. 21, 621-622, 2004.

[15] Demir, A., Kanca, F., Ozbilge, E., Numerical solution and distinguishability in time fractional parabolic equation, Bound. Val. Prblms., Article No: 142, 2015.

[16] Djrbashian, M. M., Differential Operators of fractional order and boundary value problems in the complex domain, The Gohberg Anniversary Collection, 153-172, Springer, 1989.

[17] Erdogan, A. S., Ashyralyev, A. On the second order implicit difference schemes for a right hand side identification problem, Appl.Math.Comp. 226, 212-229, 2014.

[18] Erdogan, A. S., Sazaklioglu, A.U., A note on the numerical solution of an identification problem for observing two-phase flow in capillaries, Math. Methds. Appl. Sci., 37, 23932405, 2014.

[19] Fatullayev, A. F., Numerical procedure for the simultaneous determination of unknown coefficients in a parabolic equation, Appl. Math. Comp. 164, 697-705, 2005.

[20] Francesco, M., Luchko, Y., Pagnini, G. The fundamental solution of the space-time fractional diffusion equation, Arxiv prep. cond-mat, p.0702419, 2007.

[21] Gorenflo, R., Mainardi, F., Moretti, D., Paradisi, P., Time fractional diffusion: a discrete random walk approach, Nonlinear Dynamics, 29, 129-143, 2002.

[22] Luchko, Y., Initial boundary value problems for the one dimentional time-fractional diffusion equation, Frac. Calc. Appl. Analy., 15, 141-160, 2012.

[23] Ozbilge, E., Demir,A., Analysis of the inverse problem in a time fractional parabolic equation with mixed boundary conditions, Bound.Value. Prblms., Article No:134, 2014.

[24] Ozbilge, E., Demir,A., Identification of unknown coefficient in time fractional parabolic equation with mixed boundary conditions via semigroup approach, Dyn. Syst. Appl., 24(3), 341-348, 2015.

[25] Ozbilge, E., Demir,A., Inverse problem for a time-fractional parabolic equation, Jour. of Ineq. and Appl., Article No:81, 2015.

[26] Ozbilge, E., Demir, A., Kanca, F., Ozbilge, E., Determination of the unknown source function in time fractional parabolic equation with dirichlet boundary conditions, Appl. Math. Inf. Sci., 10(1), 283-289, 2016.

[27] Plociniczak, L., Approximation of the erdelyi-kober operator with application to the timefractional porous medium equation, SIAM J. Appl. Math., 74(4), 1219-1237, 2014.

[28] Plociniczak, L., Analytical studies of a time-fractional porous medium equation. Derivation, approximation and applications, Comm. Nonlin.Sci. Num.Simul., 24(1), 169-183, 2015. 
[29] Wei, H., Chen, W., Sun, H., Li, X., A coupled method for inverse source problem of spatial fractional anomalous diffusion equations, Inv. Prblms. in Sci. Eng., 18(7), 945-956, 2010.

[30] Wei, T., Zhang, Z.Q., Reconstruction of a time-dependent source term in a time-fractional diffusion equation, Eng. Analys.Boundry. Elemts., 37(1), 23-31, 2013.

[31] Zhang, Y., Xiang, X., Inverse source problem for a fractional diffusion equation, Inv. Prblms., 27(3):035010, 2011. 